\title{
Brownian Dynamics of the Polarization of Rodlike Polyelectrolytes: Anisotropy and the Effect of Hydrodynamic Interactions
}

\author{
Jan Antosiewicz \\ Department of Biophysics, Warsaw University, 02-089 Warsaw, Poland \\ Dietmar Porschke* \\ Max Planck Institut für biophysikalische Chemie, D 37077 Göttingen, Germany \\ Received: July 2, 1996; In Final Form: March 4, $1997^{\circledR}$
}

\begin{abstract}
The polarization of rodlike polyelectrolytes has been analyzed by Brownian dynamics simulations, using a cube with a polymer in the center surrounded by counterions and byions. Electrostatic interactions both in the cube and beyond the cube were evaluated assuming periodic boundary conditions using Ewald summation. The effect of hydrodynamic interactions on the dynamics of the ions has been evaluated by modified Oseen interaction tensors. For polyions without added salt, the dipole induced in the presence of hydrodynamic interactions is smaller than in their absence for most of our simulated data, but under some conditions the effect of hydrodynamic interactions is rather small. For polyions with 40 and 60 residues, the dipole moment induced perpendicular to the rod axis, $\mu_{\perp}$, is rather close to that induced parallel to the rod axis, $\mu_{\|}$. The difference $\left(\mu_{\|}-\mu_{\perp}\right)$ is positive under most conditions, but negative values of $\left(\mu_{\|}-\mu_{\perp}\right)$ were also found in some cases. All simulated dipole moments increase with the chain length, $N ; \mu_{\perp}$ shows a linear increase with the chain length, whereas the increase of $\mu_{\|}$and $\left(\mu_{\|}-\mu_{\perp}\right)$ with $N$ is more than linear. The dipole moment $\mu_{\|}$calculated for a polyion with 40 residues in the presence of hydrodynamic interactions increases with increasing concentration of added salt, whereas in the absence of hydrodynamic interactions $\mu_{\|}$is virtually independent of the salt concentration. This result indicates that the contribution of hydrodynamic interactions to the dipole moment increases with increasing salt concentration. Electric fields induce not only dipole moments but also dissociation of ions, which is reflected by an increase of the root mean square distance $\left\langle s_{\mathrm{c}}\right\rangle^{1 / 2}$ of the counterions from the center of the cube. The increase of $\left\langle s_{\mathrm{c}}{ }^{2}\right\rangle^{1 / 2}$ computed in the presence of hydrodynamic interactions is smaller than in their absence; this increase is also smaller for perpendicular than for parallel orientation of the field vector and rod axis. The rise-time constants of the dipole moments are in the time range of a few nanoseconds and do not show any detectable dependence on the chain length. Because of the complexity of the results, which is due to the superposition of many different effects, it is not possible at the present state of the simulations to present simple equations or general rules for the anisotropy of the polarizability, e.g. as a function of the field strength or of the chain length.
\end{abstract}

\section{Introduction}

The polarization of rodlike polyelectrolytes in external electric fields is a complex process which remains a major problem for a quantitative theoretical description. ${ }^{1-22}$ Various theoretical approaches have been used, but it proves to be difficult to include all the different effects that are expected to contribute to polarization into a tractable model. The electrostatics of polarization alone is already complex enough because of the interactions of many charged species with each other and with the external electric field. However, in addition to electrostatics it is expected that there are hydrodynamic interactions between the components. According to the calculations of Fixman and Jagannathan, ${ }^{9}$ hydrodynamic interactions provide a dominant contribution to the polarizability of polyelectrolytes.

Because of the complexity of the problem, simulations of counterion polarization around rodlike polyelectrolytes by Brownian dynamics appear to be the method of choice. We have used this approach previously ${ }^{21}$ but did not include hydrodynamic interactions yet and also did not study the anisotropy of polarization. In our present contribution we describe simulations on the polarization of rodlike polyelectrolytes in the presence and in the absence of hydrodynamic interactions. According to the results, the influence of hydrodynamic interactions is very much dependent on the conditions,

\footnotetext{
${ }^{\otimes}$ Abstract published in Advance ACS Abstracts, May 1, 1997.
}

e.g. the chain length, the electric field strength, and the salt concentration. We have also compared dipole moments induced by electric fields applied parallel and the perpendicular to the long axis of rods and find that the polarizability in perpendicular direction is considerable and is almost as large as that in the parallel direction for polyions with up to 60 residues.

\section{Simulation Procedure}

Our procedure for the simulation of the dynamics of counterions and coions around a cylindrical polyion has been described in detail, ${ }^{21}$ and thus our present description is mainly devoted to the new subroutines introduced into the program. We analyze a model consisting of a single rigid cylindrical polyion with a given number of counterions and byions in a cube with side length $L$. This cube is surrounded by identical images using periodical boundary conditions. Electrostatic interactions between the polyion, counterions, and byions are evaluated using the Ewald summation technique. ${ }^{23,24}$ The components of the cube are equilibrated during an initial period of simulation in the absence of an external electric field and then polarization is studied by simulation of trajectories for counterions and byions under a uniform external electric field of strength $E$. In our previous investigation we have applied the electric field along the axis of the polyion. The extension of this procedure to simulations in electric fields directed perpendicular to the long polymer axis does not require a special 
description. However, the introduction of hydrodynamic interactions between the polyion, counterions, and byions is not trivial and thus is described in detail in the following section.

When solute particles translate and rotate in a viscous fluid at sufficiently small Reynolds numbers, the forces and torques exerted by the fluid on the particles are linear functions of their translational and angular velocities. ${ }^{25}$ The forces and the torques may be evaluated approximately on the basis of bead models of the particles, with each bead acting as a frictional center. ${ }^{26}$ These models are used in the present work.

Consider $K$ beads. First $M$ beads with radii $a_{\mathrm{p}}$ form the model of the polyion. The remaining $K-M$ beads with radii $a_{\mathrm{s}}$ represent the counterions and byions. We assume that the polyion is rigid. The Brownian motion of the system of $K$ beads in the time interval $\Delta t=t-t_{0}$ is described by the equation ${ }^{27}$

$$
\Delta \mathbf{r}=\frac{\Delta t}{k_{\mathrm{B}} T} \hat{\mathbf{Q}} \cdot \mathbf{F}+\mathbf{R}(\Delta t)
$$

In the above equation, the vectors $\mathbf{r}, \mathbf{F}$, and $\mathbf{R}$ are $3 K \times 1$ column vectors obtained by stacking the $K$ column vectors ( 3 $\times 1), \mathbf{r}_{i}, \mathbf{F}_{i}$, or $\mathbf{R}_{i}$, on top of each other. Each $i$ th vector $\mathbf{r}_{i}, \mathbf{F}_{i}$, $\mathbf{R}_{i}$ describes net displacement, experienced force, and random displacement for each of $K$ beads. The matrix $\hat{\mathbf{Q}}$ is a symmetric $3 K \times 3 K$ supermatrix constructed from hydrodynamic interaction $3 \times 3 \hat{\mathbf{D}}_{i j}$ tensors between beads $i$ and $j$. Thus

$$
\hat{\mathbf{Q}}=\left(\begin{array}{llll}
\hat{\mathbf{D}}_{11} & \hat{\mathbf{D}}_{12} & \ldots & \hat{\mathbf{D}}_{1 K} \\
\hat{\mathbf{D}}_{21} & \hat{\mathbf{D}}_{22} & \ldots & \hat{\mathbf{D}}_{2 K} \\
\vdots & \vdots & \ddots & \vdots \\
\hat{\mathbf{D}}_{K 1} & \hat{\mathbf{D}}_{K 2} & \ldots & \hat{\mathbf{D}}_{K K}
\end{array}\right)
$$

and $^{26,28,29}$

$$
\begin{aligned}
& \hat{\mathbf{D}}_{i j}= \\
& \begin{cases}\frac{k_{\mathrm{B}} T}{6 \pi \eta a_{i}} \hat{\mathbf{I}} \delta_{i j}, & \text { if } i=j \\
\frac{k_{\mathrm{B}} T}{8 \pi \eta r_{i j}}\left[\left(\hat{\mathbf{I}}-\frac{\mathbf{r}_{i j} \mathbf{r}_{i j}}{r_{i j}^{2}}\right)+\frac{a_{i}^{2}+a_{j}^{2}}{r_{i j}^{2}}\left(\frac{1}{3} \hat{\mathbf{I}}-\frac{\mathbf{r}_{i j} \mathbf{r}_{i j}}{r_{i j}^{2}}\right)\right], & \begin{array}{c}
\text { if } i \neq j, \\
r_{i j} \geq a_{i}+a_{j} \\
\frac{k_{\mathrm{B}} T}{6 \pi \eta a_{i}}\left[\left(1-\frac{9}{32} \frac{\mathbf{r}_{i j}}{a_{i}}\right) \hat{\mathbf{I}}+\frac{3}{32} \frac{\mathbf{r}_{i j} \mathbf{r}_{i j}}{a_{i} r_{i j}}\right],
\end{array} \\
\begin{array}{c}
\text { if } i \neq j, a_{i}=a_{j} \\
r_{j j}<2 a_{i}
\end{array}\end{cases}
\end{aligned}
$$

In eq $3, a_{i}$ is the radius of $i$ th bead, $r_{i j}$ is the distance between beads $i$ and $j$, and $\eta$ is the viscosity of the solvent. Matrix $\hat{\mathbf{Q}}$ and vector $\mathbf{F}$ are evaluated at the beginning of time step $\Delta t$. We are using a modified Oseen hydrodynamic interaction tensor for both nonoverlapping beads of arbitrary radii and for overlapping beads of the same radii. There is no expression available for overlapping beads of unequal radii at present. Consequently, all beads for the polyion should have the same radius (because in our model they overlap), and all beads of the counterions and byions should also have the same radius (because we do not forbid their overlap; when they overlap we set electrostatic interaction between them to zero; thus we avoid infinite attraction forces between oppositely charged small mobile ions). For the hydrodynamic interactions between small ions and the polyion, we need the HI tensors only for nonoverlapping beads, because during simulation polyion and ion beads are not allowed to overlap.
The vector $\mathbf{R}$ representing random step of all beads is obtained from

$$
\mathbf{R}=\hat{\mathbf{S}} \cdot \mathbf{X}
$$

where the $3 K \times 3 K$ tensor $\hat{\mathbf{S}}$ is derived from the tensor $\hat{\mathbf{Q}}$ according to

$$
\hat{\mathbf{Q}}=\hat{\mathbf{S}} \cdot \hat{\mathbf{S}}
$$

and $\mathbf{X}$ is a column with $3 K$ random Gaussian numbers with zero mean and $2 \Delta t$ variance. Thus finally we may represent Brownian motion of a set of $K$ beads by the following form of eq 1:

$$
\begin{aligned}
& \Delta\left(\begin{array}{l}
\mathbf{r}_{1} \\
\mathbf{r}_{2} \\
\vdots \\
\mathbf{r}_{K}
\end{array}\right)=\frac{\Delta t}{k_{\mathrm{B}} T}\left(\begin{array}{llll}
\hat{\mathbf{D}}_{11} & \hat{\mathbf{D}}_{11} & \ldots & \hat{\mathbf{D}}_{1 K} \\
\hat{\mathbf{D}}_{21} & \hat{\mathbf{D}}_{22} & \ldots & \hat{\mathbf{D}}_{2 K} \\
\vdots & \vdots & \ddots & \vdots \\
\hat{\mathbf{D}}_{K 1} & \hat{\mathbf{D}}_{K 2} & \ldots & \hat{\mathbf{D}}_{K K}
\end{array}\right) \cdot\left(\begin{array}{l}
\mathbf{F}_{1} \\
\mathbf{F}_{2} \\
\vdots \\
\mathbf{F}_{K}
\end{array}\right)+ \\
& \\
&\left(\begin{array}{llll}
\hat{\mathbf{S}}_{11} & \hat{\mathbf{S}}_{12} & \ldots & \hat{\mathbf{S}}_{1 K} \\
\hat{\mathbf{S}}_{21} & \hat{\mathbf{S}}_{22} & \ldots & \hat{\mathbf{S}}_{2 K} \\
\vdots & \vdots & \ddots & \vdots \\
\hat{\mathbf{S}}_{K 1} & \hat{\mathbf{S}}_{K} & \ldots & \hat{\mathbf{S}}_{K K}
\end{array}\right) \cdot\left(\begin{array}{l}
\mathbf{X}_{1} \\
\mathbf{X}_{2} \\
\vdots \\
\mathbf{X}_{K}
\end{array}\right)
\end{aligned}
$$

Because we assume a rigid model of the polyion, we do not need to consider motion of its $M$ beads separately. Thus we can split eq 6 into two parts, one for motion of the whole polyion and the second for motions of counterions and byions. Doing this, in the current version of our program we neglect rotational motion of the polyion. We calculate the translational diffusion tensor of the bead model of the polyion $\hat{\mathbf{T}}_{\mathrm{P}}$, according to methods described elsewhere. ${ }^{26,30,31}$ We define the coordinates of beads of the polyion in the eigen axes of its translational diffusion tensor (this is straightforward because of assumed cylindrical symmetry of the polyion); thus the translational diffusion tensor of the polyion is diagonal:

$$
\hat{\mathbf{T}}_{\mathrm{P}}=\left(\begin{array}{lll}
\mathrm{T}_{\mathrm{xx}} & 0 & 0 \\
0 & T_{\mathrm{yy}} & 0 \\
0 & 0 & T_{\mathrm{zz}}
\end{array}\right)
$$

Translational random steps of the polyion, $\mathbf{R}_{\mathrm{P}}$, are obtained by three independent Gaussian random numbers with zero mean value and variances $2 T_{i i} \Delta t$ for the $i$ th axis. Thus movement of the polyion itself is determined by

$$
\Delta \mathbf{r}_{\mathrm{P}}=\frac{\Delta t}{k_{\mathrm{B}} T} \hat{\mathbf{T}}_{\mathrm{P}} \cdot \mathbf{F}_{\mathrm{P}}+\mathbf{R}_{\mathrm{P}}(\Delta t)
$$

where $\mathbf{F}_{\mathrm{P}}$ is the total electrostatic force exerted on the polyion. The Brownian motion of $K-M$ beads of mobile ions is determined by

$$
\begin{aligned}
& \Delta\left(\begin{array}{l}
\mathbf{r}_{M+1} \\
\mathbf{r}_{M+2} \\
\vdots \\
\mathbf{r}_{K}
\end{array}\right)=\frac{\Delta t}{k_{\mathrm{B}} T}\left(\begin{array}{llll}
\hat{\mathbf{D}}_{M+1,1} & \hat{\mathbf{D}}_{M+1,2} & \ldots & \hat{\mathbf{D}}_{M+1, K} \\
\hat{\mathbf{D}}_{M+2,1} & \hat{\mathbf{D}}_{M+2,2} & \ldots & \hat{\mathbf{D}}_{M+2, K} \\
\vdots & \vdots & \ddots & \vdots \\
\hat{\mathbf{D}}_{K, 1} & \hat{\mathbf{D}}_{K, 2} & \ldots & \hat{\mathbf{D}}_{K, K}
\end{array}\right) \cdot\left(\begin{array}{l}
\mathbf{F}_{1} \\
\mathbf{F}_{2} \\
\vdots \\
\mathbf{F}_{K}
\end{array}\right)+ \\
& \left(\begin{array}{llll}
\hat{\mathbf{S}}_{M+1,1} & \hat{\mathbf{S}}_{M+1,2} & \ldots & \hat{\mathbf{S}}_{M+1, K} \\
\hat{\mathbf{S}}_{M+2,1} & \hat{\mathbf{S}}_{M+2,2} & \ldots & \hat{\mathbf{S}}_{M+2, K} \\
\vdots & \vdots & \ddots & \vdots \\
\hat{\mathbf{S}}_{K, 1} & \hat{\mathbf{S}}_{K, 2} & \ldots & \hat{\mathbf{S}}_{K, K}
\end{array}\right) \cdot\left(\begin{array}{l}
\mathbf{X}_{1} \\
\mathbf{X}_{2} \\
\vdots \\
\mathbf{X}_{K}
\end{array}\right)
\end{aligned}
$$

where $\mathbf{F}_{i}(i=1, \ldots, K)$ are electrostatic forces experienced by 
individual beads and $\boldsymbol{X}_{i}(i=1, \ldots, K)$ are random Gaussian numbers with variance $2 \Delta t$.

After moving the polyion according to eq 8 and moving all mobile small ions according to eq 9 , the final positions of the mobile ions are corrected for the movement of the polyion and the next step in Brownian dynamics simulation starts. Thus the results of our simulation are motions of mobile ions in the coordinate system fixed to the polyion.

The polymer is represented by a cylinder with a line of $N$ negative unit charges with a constant spacing parameter $b$ located on the symmetry axis (note that the number of charges of the polymer $N$ is larger than the number of beads $M$ used for the hydrodynamic model representing the polymer). The radius of the cylinder is $a$ and the length is $l=(N-1) b+2 a$. In all our simulations we use $b=0.17 \mathrm{~nm}$ and $a=0.5 \mathrm{~nm}$; the charge spacing corresponds to that of double-helical DNA; that is, two charges correspond to one base pair. The ions are assumed to be in a liquid solvent; we use a relative dielectric constant $\epsilon_{\mathrm{r}}=$ 80.2 and a viscosity $1.002 \times 10^{-3} \mathrm{~kg} \mathrm{~m}^{-1} \mathrm{~s}^{-1}$ corresponding to an aqueous solution at $20{ }^{\circ} \mathrm{C}$. The translational diffusion coefficient of counterions and byions is $1.16 \times 10^{-9} \mathrm{~m}^{2} \mathrm{~s}^{-1}$.

For each transient we calculate at given sampling intervals the dipole vector of the box

$$
\vec{\mu}=\sum_{i=1}^{\mathrm{NI}} q_{i} \vec{r}_{i}
$$

and the root mean square distance of the counterions from the center of the box

$$
\left\langle s_{\mathrm{c}}{ }^{2}\right\rangle^{1 / 2}=\left(\sum_{i=1}^{\mathrm{NC}} r_{i}^{2}\right)^{1 / 2}
$$

where NI is the number of ions, including all ions with both positive and negative charge, and $\mathrm{NC}$ is the number of counterions, corresponding to the ions with positive charge.

We have also collected data on the motion of the polymer and calculated the electrophoretic mobility (the distances moved by the polymer in the direction of the field during each time step were divided by the time increments and the resulting values were averaged). Individual transients were accumulated and the results averaged, until the signal to noise ratio was sufficient. The averaged number of individual transients was in the range between 100 and 1000; usually higher numbers of individual transients were simulated for the short polymers.

\section{Results}

As described in the previous publication, ${ }^{21}$ application of an electric field to a box containing a polyelectrolyte induces a dipole moment in the direction of the vector of the applied field with rise-time constants in the nanosecond time range. At low field strengths the rise of the dipole moment can be described by single exponentials, whereas two exponentials $\tau_{1}^{\mathrm{r}}$ and $\tau_{2}^{\mathrm{r}}$ are required at higher field strengths (cf. Figure 1). The maximum of the dipole moment appearing shortly after application of the electric field seems to be due to field-induced dissociation of counterions: $:^{32-36}$ the counterions, which are dissociated from the polymer, move away from the polymer and thus contribute to the dipole moment. In order to keep a constant number of particles in the box during the simulation, ions moving out of the box at one boundary plane are introduced at the opposite boundary of the box. Due to this operation, the overshoot effect is turned into an undershoot effect after a characteristic time. In some cases this effect leads to an oscillation phenomenon;
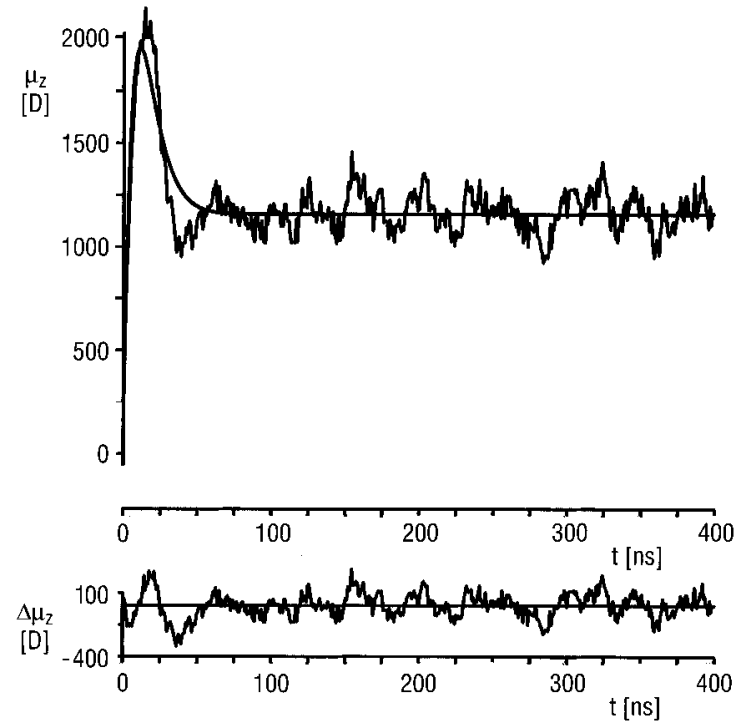

Figure 1. Dipole moment $\mu_{\|}$induced by an electric field of $E=50$ $\mathrm{kV} / \mathrm{cm}$ as a function of time $t$ in a $40 \times 40 \times 40 \mathrm{~nm}$ cube with a polymer of 40 residues and 10 byions. The line without noise represents a least squares fit with two exponentials $\left(\tau_{1}^{\mathrm{r}}=7.9 \mathrm{~ns}, \tau_{2}^{\mathrm{r}}=8.2 \mathrm{~ns}\right.$, $\Delta \mu_{1}^{\mathrm{r}}=80.51 \mathrm{kD}, \Delta \mu_{2}^{\mathrm{r}}=-79.35 \mathrm{kD}$, average of 1750 individual transients). The lower panel shows the residuals of the fit as a function of time.
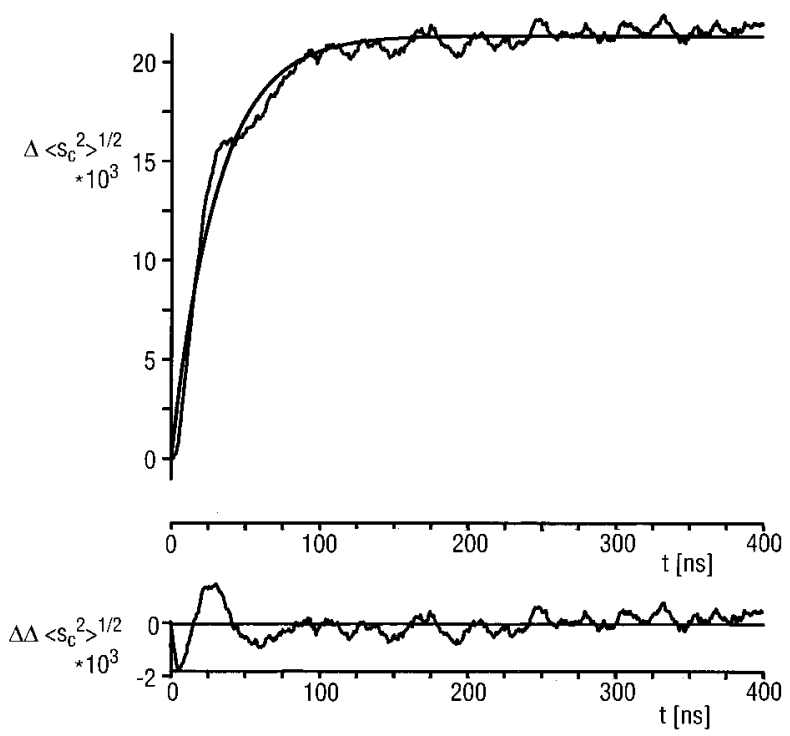

Figure 2. Change of the root mean square distances $\left\langle s_{\mathrm{c}}^{2}\right\rangle^{1 / 2}$ of counterions and induced by an electric field $E=50 \mathrm{kV} / \mathrm{cm}$ as a function of time $t$ in a $40 \times 40 \times 40 \mathrm{~nm}$ cube with a polymer of 40 residues and 10 byions. The line without noise represents a least squares fit with a single exponential $\left(\tau=30 \mathrm{~ns},\left(\Delta\left\langle s_{\mathrm{c}}{ }^{2}\right\rangle^{1 / 2}\right)_{\infty}=2.13 \times 10^{-3}\right.$, average of 1750 individual transients). The lower panel shows the residuals of the fit as a function of time.

in all cases the over-/undershoot effects cancel out owing to Brownian motion after some time of about 100 ns. These effects appear both in the presence and in the absence of hydrodynamic interactions and also for electric fields applied both parallel and perpendicular to the polymer axis.

The field-induced dissociation of ions from the ion atmosphere of the polymer has been documented by calculation of root mean square distances of counterions and byions $\left\langle s_{\mathrm{c}}{ }^{2}\right\rangle^{1 / 2}$. This parameter increases after application of electric fields within a time period of about 100 ns (cf. Figure 2). In most cases the increase of $\left\langle s_{\mathrm{c}}{ }^{2}\right\rangle^{1 / 2}$ as a function of time $t$ obtained for high field strengths requires two exponentials $\tau_{1}^{\mathrm{s}}$ and $\tau_{2}^{\mathrm{s}}$ for a satisfactory fit. The signal to noise ratio of the data obtained 


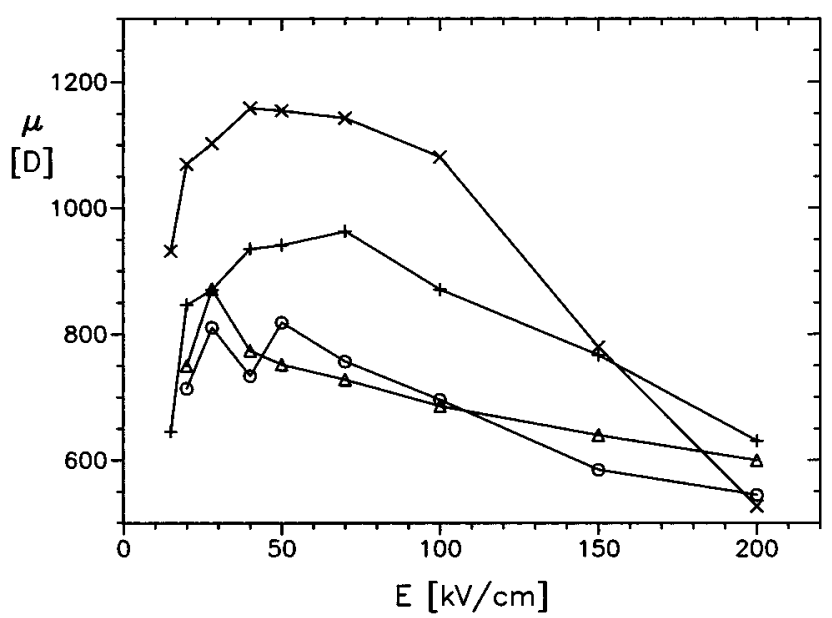

Figure 3. Stationary values of the dipole moments $\mu$ induced for a polymer with 40 residues in a $40 \times 40 \times 40 \mathrm{~nm}$ cube as a function of the electric field strength $E$. With hydrodynamic interactions: electric field parallel $(\triangle)$ and perpendicular $(\bigcirc)$ to the polymer axis. Without hydrodynamic interactions: electric field parallel $(x)$ and perpendicular $(+)$ to the polymer axis.

at low field strength is usually lower, and thus these data cannot be fitted by more than single exponentials. The time constant $\tau_{2}^{\mathrm{s}}$ obtained from the $\left\langle s_{\mathrm{c}}{ }^{2}\right\rangle^{1 / 2}$ data is clearly larger than $\tau_{2}^{\mathrm{r}}$ obtained from the rise curves of the dipole moments. It must be expected that the field-induced response of a polyelectrolyte cannot be described in terms of a few normal modes. Thus the time constants obtained from the simulations at the present level of accuracy simply represent averages, and the weighting factors for dipole transients and for $\left\langle s_{\mathrm{c}}{ }^{2}\right\rangle^{1 / 2}$ transients appear to be different.

The stationary values of both the dipoles and the root mean square distances have been evaluated by least squares exponential fits of the averaged transients, as shown in the examples of Figures 1 and 2. Because of limitations in the available computer time, the number of transients used for averaging had to be limited, and thus also the signal to noise ratio remained limited. The simulations involving hydrodynamic interactions required particularly large amounts of computer time. In spite of limitations resulting from these boundary conditions, some new information may be derived from the available data.

As should be expected the stationary values of the dipole moments increase with increasing field strength $E$. However, the dipole moments approach saturation already at field strengths $E \approx 50 \mathrm{kV} / \mathrm{cm}$ for polymers with both 40 and 60 charged residues (cf. Figures 3 and 4). Moreover, the dipole moments decrease with increasing $E$ at $E>50 \mathrm{kV} / \mathrm{cm}$. These effects appear-with some minor variation-in all of the simulated data sets and do not depend on the direction of the applied field with respect to the polymer axis and also do not depend on the presence or absence of hydrodynamic interactions. It is remarkable that for polymers with 40 and 60 charged residues the dipole induced perpendicular to the polymer axis is almost as large as the dipole induced parallel to this axis. Furthermore, the data obtained for polyions without added salt show that the dipoles induced in the presence of hydrodynamic interactions are smaller than those in their absence in most cases. However, in the range of relatively small electric field strengths $E<30$ $\mathrm{kV} / \mathrm{cm}$ it is not possible yet to derive definite conclusions, because in this range the induced dipole moments are not accurate enough and differences are within the noise level.

Experimental data do not provide the individual dipole components but only the difference $\left(\mu_{\|}-\mu_{\perp}\right)$. The $\left(\mu_{\|}-\mu_{\perp}\right)$ values simulated for the polymer with 40 residues are close to

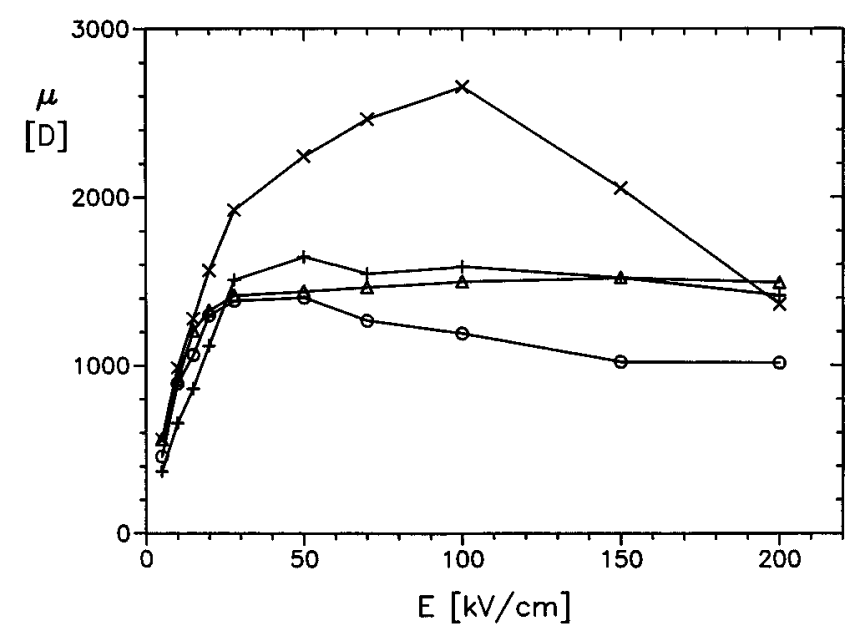

Figure 4. Stationary values of the dipole moments $\mu$ induced for a polymer with 60 residues in a $40 \times 40 \times 40 \mathrm{~nm}$ cube. With hydrodynamic interactions: electric field parallel to polymer axis $(\triangle)$ and perpendicular to polymer axis $(O)$. Without hydrodynamic interactions: electric field parallel to polymer axis $(x)$ and perpendicular to polymer axis $(+)$.



Figure 5. Stationary values of the difference $\mu_{\|}-\mu_{\perp}$ between the dipole moments induced parallel and perpendicular to the polymer axis; in the presence of hydrodynamic interactions for polymers with $40(\mathrm{O})$ and with $60(\triangle)$ residues, in the absence of hydrodynamic interactions for polymers with $40(+)$ and $60(\times)$ residues. All simulations in $40 \times$ $40 \times 40 \mathrm{~nm}$ cubes.

the zero level (cf. Figure 5), but these data indicate that $\left(\mu_{\|}-\right.$ $\mu_{\perp}$ ) is larger in the absence than in the presence of hydrodynamic interactions except for the highest field strengths, $E \geq 150 \mathrm{kV} /$ $\mathrm{cm}$. A closely corresponding result is found for the polymer with 60 residues, the only difference being that in this case the values tend to be higher.

The chain length dependence of the induced dipole moment is of particular interest. For a comparison of data simulated for different chain lengths the conditions used in the simulations should be equivalent. Using the arguments discussed previously, ${ }^{21}$ we have simulated dipole moments for different chain lengths under conditions where the distance between the terminal residues of the polymer and the cube boundary is constant. For this purpose the cube size is increased with increasing length of the polymer. Furthermore the concentration of cations in the box should be constant. The dipole moments simulated under these conditions are compiled in Table 1. All the dipole moments increase with increasing chain length $N$. The $\mu_{\perp}$ values show a linear increase with $N$, whereas the increase of $\mu_{\|}$and of $\left(\mu_{\|}-\mu_{\perp}\right)$ with $N$ is more than linear, but a simple function for this increase cannot be derived yet with a sufficient reliability at the present state of the simulations. According to the 
TABLE 1: Stationary Values of the Induced Dipole Moments $\boldsymbol{\mu}$ and of the Changes of the Root Mean Square Distances $\left(\Delta\left\langle s_{\mathrm{c}}{ }^{2}\right\rangle^{1 / 2}\right)_{\infty}{ }^{a}$

\begin{tabular}{|c|c|c|c|c|c|c|}
\hline \multirow[b]{2}{*}{$E$} & \multirow[b]{2}{*}{$N$} & \multicolumn{3}{|c|}{ dipole moment [D] } & \multicolumn{2}{|c|}{$\left[\left(\Delta\left\langle s_{\mathrm{c}}{ }^{2}\right\rangle^{1 / 2}\right)_{\infty}\right] \times 10^{3}$} \\
\hline & & $\mu_{\|}$ & $\mu_{\perp}$ & $\mu_{\|}-\mu_{\perp}$ & II & $\perp$ \\
\hline \multirow[t]{4}{*}{50} & 40 & 1161 & 924 & 237 & 21.4 & 15.0 \\
\hline & 60 & 1663 & 1355 & 308 & 31.1 & 16.9 \\
\hline & 80 & 2283 & 1940 & 343 & 31.1 & 20.0 \\
\hline & 98 & 3238 & 2508 & 730 & 32.0 & 22.0 \\
\hline \multirow{4}{*}{100} & 40 & 1026 & 840 & 186 & 37.7 & 27.2 \\
\hline & 60 & 1598 & 1284 & 314 & 44.2 & 33.0 \\
\hline & 80 & 2578 & 1675 & 903 & 50.9 & 34.6 \\
\hline & 98 & 3917 & 2148 & 1769 & 54.1 & 37.6 \\
\hline
\end{tabular}

${ }^{a}$ The components induced by electric fields parallel and perpendicular to the polymer axis are indicated by the indices $\|$ and $\perp$, respectively. The electric field strength $E$ is given in $[\mathrm{kV} / \mathrm{cm}]$, the chain length $N$ is the number of charged residues. Some details of the simulations: the spacing of the charged residues is $0.17 \mathrm{~nm}$; in the case of the polymer with 40 residues the cube was $40 \times 40 \times 40 \mathrm{~nm}$ and contained 10 byions; the cube size was increased with the chain length, such that there was a distance of $16.6 \mathrm{~nm}$ between the ends of the polymer and the cube boundary; in all cases the number of cations in the cube corresponded to a concentration of $1.3 \mathrm{mM}$. Estimated accuracy: $\mu_{\|}$and $\mu_{\perp} \pm 10 \% ;\left(\mu_{\|}-\mu_{\perp}\right) \pm 20 \% ;\left(\Delta\left\langle s_{\mathrm{c}}^{2}\right\rangle^{1 / 2}\right)_{\infty} \pm 5 \%$.

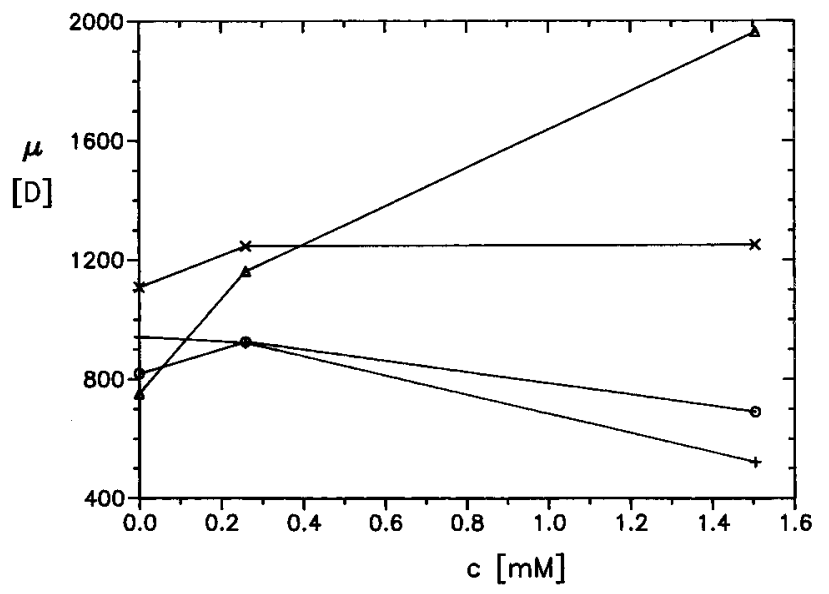

Figure 6. Stationary values of the dipole moments $\mu$ induced for a polymer with 40 residues as a function of the salt concentration $c$ in a $40 \times 40 \times 40 \mathrm{~nm}$ cube at an electric field strength $E=50 \mathrm{kV} / \mathrm{cm}$. With hydrodynamic interactions: electric field parallel $(\triangle)$ and perpendicular $(O)$ to the polymer axis. Without hydrodynamic interactions: electric field parallel $(x)$ and perpendicular $(+)$ to the polymer axis.

available data, there is a stronger increase of $\mu_{\|}$and of $\left(\mu_{\|}-\right.$ $\left.\mu_{\perp}\right)$ with $N$ at 100 than at $50 \mathrm{kV} / \mathrm{cm}$.

It should be noted that the dipole moments shown in Figures 3 and 4 for $N=40$ and 60 are not identical with those given in Table 1. The data shown in Figures 3 and 4 were simulated in $40 \times 40 \times 40 \mathrm{~nm}$ cubes without byions; the data shown in Table 1 for $N=40$ were also simulated in a $40 \times 40 \times 40 \mathrm{~nm}$ cube, but this cube contained 10 byions in addition to the polyion; the data shown in Table 1 for $N=60$ were simulated in a $43.4 \times 43.4 \times 43.4 \mathrm{~nm}$ cube containing four byions. A comparison of the data simulated for $N=40$ indicates that addition of byions leads to an increase of the dipole moment. A summary of data computed on the salt dependence is given in Figure 6. The dipole moment $\mu_{\|}$simulated in the presence of hydrodynamic interactions clearly increases with the salt concentration $c$, whereas the corresponding value $\mu_{\perp}$ decreases with increasing $c$. In the absence of hydrodynamic interactions, the dipole moments are virtually independent of the salt concentration. These data indicate that the influence of hydrodynamic interactions increases with increasing salt concentration.

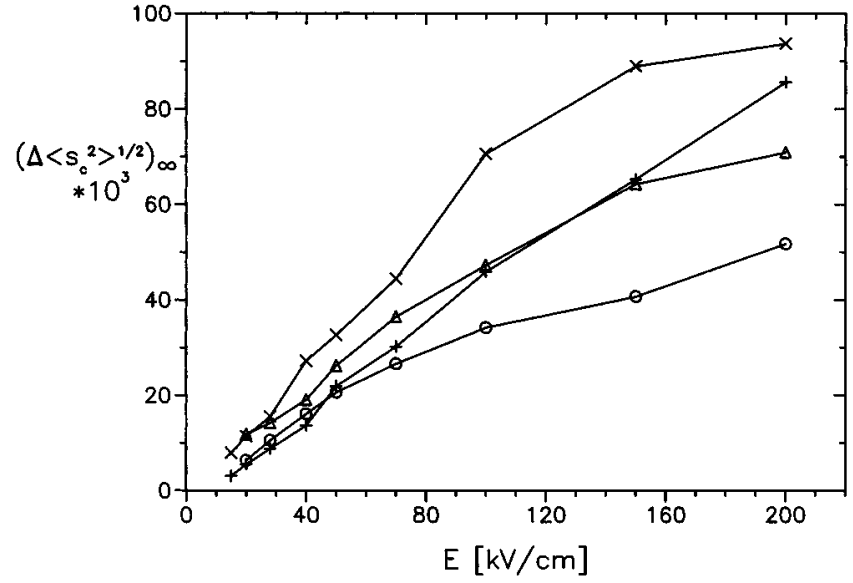

Figure 7. Stationary values of the increase of the root mean square distance $\left(\Delta\left\langle s_{\mathrm{c}}{ }^{2}\right\rangle^{1 / 2}\right)_{\infty}$ as a function of the electric field strength $E$ for a polymer with 40 residues in a $40 \times 40 \times 40 \mathrm{~nm}$ cube; in the presence of hydrodynamic interactions with the electric field parallel to the polymer axis $(\triangle)$ and perpendicular to the polymer axis) $(O)$; in the absence of hydrodynamic interactions with the electric field parallel to the polymer axis $(x)$ and perpendicular to the polymer axis $(+)$.

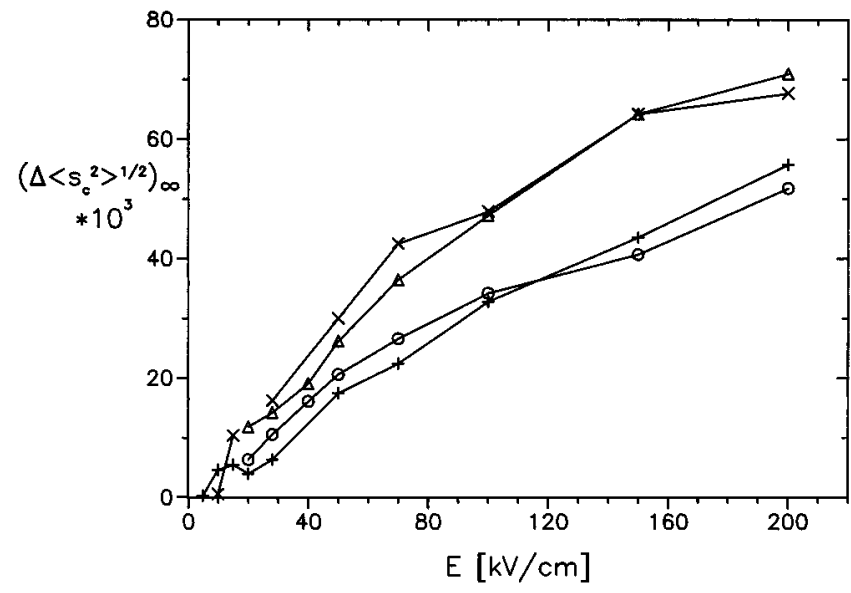

Figure 8. Stationary values of the increase of the root mean square distance $\left(\Delta\left\langle s_{\mathrm{c}}^{2}\right\rangle^{1 / 2}\right)_{\infty}$ as a function of the electric field strength $E$ in the presence of hydrodynamic interawctions. For a polymer with 40 residues: electric field parallel to the polymer axis $(\triangle)$ and perpendicular to the axis $(O)$. For a polymer with 60 residues: electric field parallel to the polymer axis $(\times)$ and perpendicular to the axis $(+)$. All simulations in $40 \times 40 \times 40 \mathrm{~nm}$ cubes.

The rather complex dependence of the dipole moments on the various parameters, in particular on the electric field strength, is clearly due to superposition of different effects. One of these effects is the field induced dissociation of ions from the ion atmosphere of the polymer. ${ }^{32-36}$ This process has been documented in our simulations by recording root mean square distances $\left\langle s_{\mathrm{c}}{ }^{2}\right\rangle^{1 / 2}$ of counterions and byions from the center of the cube. As shown in Figures 7 and 8, the stationary values $\left(\Delta\left\langle s_{\mathrm{c}}^{2}\right\rangle^{1 / 2}\right)_{\infty}$ increase with the electric field strength $E$. According to the data given in Figure 8 , there appears to be a relatively small increase of $\left(\Delta\left\langle s_{\mathrm{c}}{ }^{2}\right\rangle^{1 / 2}\right)_{\infty}$ when the chain length is increased from 40 to 60 residues. The data compiled in Table 1 over a wider range of chain lengths $N$ demonstrate more clearly that $\left(\Delta\left\langle s_{\mathrm{c}}{ }^{2}\right\rangle^{1 / 2}\right)_{\infty}$ increases with $N$. The values $\left(\Delta\left\langle{s_{\mathrm{c}}}^{2}\right\rangle^{1 / 2}\right)_{\infty}$ are smaller in the presence of hydrodynamic interactions than in their absence; furthermore $\left(\Delta\left\langle s_{\mathrm{c}}{ }^{2}\right\rangle^{1 / 2}\right)_{\infty}$ is smaller in the perpendicular than in the parallel direction of the electric field relative to the polymer axis.

The simulations have also been used to obtain information on the electrophoretic mobility $u$ of the polymer. As shown in Figure 9 for the example of the polymer with 60 residues, the 


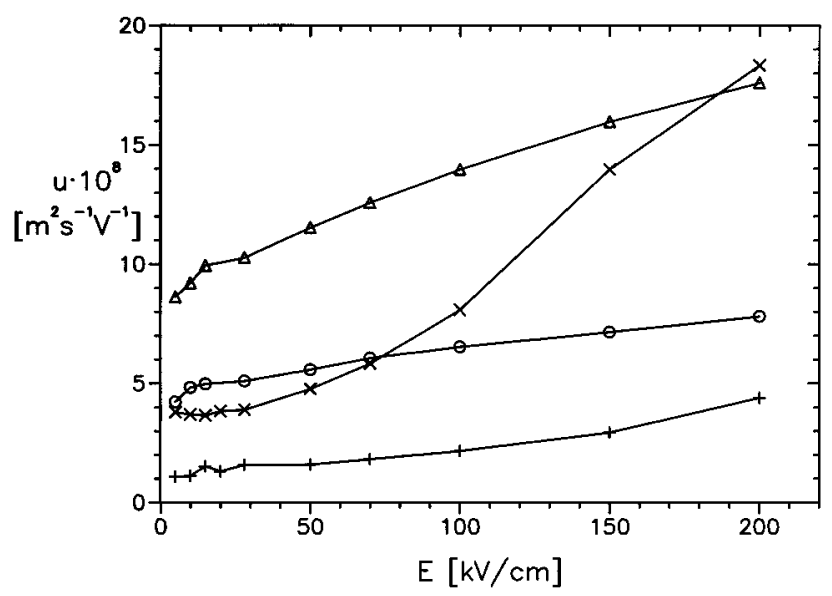

Figure 9. Electrophoretic mobilities $u$ as a function of the electric field strength $E$ for a polymer with 60 residues in a $40 \times 40 \times 40 \mathrm{~nm}$ cube; in the presence of hydrodynamic interactions with the electric field parallel to the polymer axis $(\triangle)$ and perpendicular to the axis $(O)$; in the absence of hydrodynamic interactions with the electric field parallel to the polymer axis $(\times)$ and perpendicular to the axis $(+)$. The number of averaged transients ranges from 300 at the lowest to 60 at the highest field strength. The double standard deviation, corresponding to the $95 \%$ confidence level, is $\pm 5 \times 10^{-3} \mathrm{~m} / \mathrm{s}$ for the velocities, virtually independent of the electric field strength; thus, the corresponding values for the electrophoretic mobilities decrease linearly with increasing electric field strength.

TABLE 2: Time Constants Obtained from Rise Curves of the Dipole Moments and of the Root Mean Square Distances $^{a}$

\begin{tabular}{|c|c|c|c|c|c|c|c|c|c|}
\hline \multirow[b]{3}{*}{ E } & \multirow[b]{3}{*}{$N$} & \multicolumn{4}{|c|}{ dipole rise times } & \multicolumn{4}{|c|}{$\left(\Delta\left\langle s_{\mathrm{c}}^{2}\right\rangle^{1 / 2}\right)$ rise times } \\
\hline & & \multicolumn{2}{|c|}{ II } & \multicolumn{2}{|c|}{$\perp$} & \multicolumn{2}{|c|}{ II } & \multicolumn{2}{|c|}{$\perp$} \\
\hline & & $\tau_{1}^{\mathrm{r}}$ & $\tau_{2}^{\mathrm{r}}$ & $\tau_{1}^{\mathrm{r}}$ & $\tau_{2}^{\mathrm{r}}$ & $\tau_{1}^{\mathrm{s}}$ & $\tau_{2}^{\mathrm{s}}$ & $\tau_{1}^{\mathrm{s}}$ & $\tau_{2}^{\mathrm{s}}$ \\
\hline \multirow[t]{4}{*}{50} & 40 & 7.9 & 8.2 & 11.6 & 11.7 & & 30 & & 24 \\
\hline & 60 & 7.6 & 7.9 & 12.6 & 12.8 & & 38 & & 24 \\
\hline & 80 & 8.0 & 8.2 & 13.6 & 13.8 & & 35 & & 25 \\
\hline & 98 & 8.9 & 9.2 & 15.3 & 15.7 & & 38 & & 39 \\
\hline \multirow[t]{4}{*}{100} & 40 & 4.4 & 4.4 & 5.8 & 5.9 & 6.7 & 39 & 13 & 48 \\
\hline & 60 & 4.0 & 4.2 & 6.1 & 6.3 & 5.3 & 35 & 10 & 40 \\
\hline & 80 & 3.7 & 3.8 & 7.0 & 7.2 & 5.9 & 48 & 1 & 23 \\
\hline & 98 & 3.8 & 4.0 & 7.6 & 7.9 & 6.0 & 57 & 13 & 48 \\
\hline
\end{tabular}

${ }^{a}$ The time constants obtained from simulations with the electric field parallel and perpendicular to the polymer axis are indicated by II and $\perp$, respectively. All time constants are given in ns. Details of the simulations as described in the footnote of Table 1. Estimated accuracy: $\pm 10 \%$.

u-values increase with increasing field strengths $E$. This increase is much larger for the mobility in the direction of the polymer axis than in the perpendicular direction. Is is very likely that the increase of the $u$ values with $E$ is due to fieldinduced dissociation of counterions from the ion atmosphere of the polymer. The electrophoretic mobility is clearly reduced by hydrodynamic interactions.

A main advantage of the Brownian dynamics approach is the fact that the simulations also provide time constants. The compilation in Table 2 demonstrates that the time constants both for the dipole rise curves and for the increase of the root mean square distance are independent of the chain length, within the limits of the simulation accuracy. This result is remarkable because most theories predict a very strong dependence on the chain length. The absence of any detectable chain length dependence in the simulated data is in agreement with experimental data derived from dichroism rise $\operatorname{curves}^{37}$ (cf. the Discussion section). Furthermore, the simulated data demon- strate a decrease of the dipole rise times with increasing field strength. Finally, the time constants $\tau_{2}^{\mathrm{s}}$ obtained from the increase of the root mean square distances are clearly larger than the values $\tau_{2}^{\mathrm{r}}$ obtained from the dipole rise curves.

The time constants in Table 2 have been collected under conditions where the transients require two exponentials, as the dipole moments pass a maximum before arriving at the stationary value. According to the interpretation of this overshoot effect given above, it must be expected that the time constants are affected by the size of the box used in the simulation. In fact the time constants tend to increase with the box size, but this dependence is usually superimposed by other effects. In general the overshoot effect decreases with decreasing field strength and with increasing salt concentration: this is due to the effect of electrophoretic motion relative to that resulting from Brownian motion of the particles. The time constants presented in Table 2 for the electric field strength of $50 \mathrm{kV} / \mathrm{cm}$ are at the limit where the influence of the box size is already relatively small, whereas the time constants at 100 $\mathrm{kV} / \mathrm{cm}$ are more strongly influenced by the box size. A clear separation of the influence of the box size on the time constants is difficult, because of the superposition of different effects: an increase of the box size around a given polyion leads to a decrease of the ion concentration; if this decrease is compensated by addition of salt, the dipole moment and its rise is affected by the different ratio of counterions to coions (cf. above).

\section{Discussion}

Theoretical models for the polarization of rodlike polyelectrolytes have been developed by many different authors, but the available models do not include all the different effects expected to occur upon application of electric fields. This is clearly due to the difficulties associated with an analytical treatment of the different effects and of their coupling with each other. Obviously the Brownian dynamics approach used in our present investigation cannot be as satisfactory as a complete analytical treatment, but in the absence of a complete theory there is hardly any alternative to Brownian dynamics simulations.

In the present investigation we have extended our previous simulations by consideration of hydrodynamic interactions. The results clearly demonstrate that the hydrodynamic interactions influence the induced dipole moments. According to the data obtained from our present simulations, the contribution resulting from hydrodynamic interactions remains rather small for polyions without added salt, but is considerable in the presence of salt. The first calculations on "convective polarization" were reported by Fixman and Jagannathan. ${ }^{9}$ They concluded that corrections due to hydrodynamic interactions are "extremely large", but were also cautious enough to mention that these corrections "will probably remain quite unreliable for some time". In view of the problems described by Fixman and Jagannathan ("primitive study of even the first correction"), it does not make much sense to compare the results in detail. We believe that the ingredients of our simulations are reasonable, because all the parameters used in the calculations are either directly derived from experimental data or derived from models that are under sufficient control by experimental data (e.g. the diffusion coefficients of the polymers). The tensors used for the description of hydrodynamic interactions proved to be correct in bead model simulations of the hydrodynamic parameters of many different macromolecules..$^{30,31,38}$

Our simulations demonstrate that for polyions of 40 and 60 residues without added salt the dipole moments induced parallel to the polymer axis, $\mu_{\|}$, are very close to those induced 
perpendicular to this axis, $\mu_{\perp}$, both in the presence and in the absence of hydrodynamic interactions. However, $\mu_{\perp}$ shows a linear increase with the chain length $N$, whereas the increase of $\mu_{\|}$with $N$ is more than linear. Apparently the increase of $\mu_{\|}-$ $\mu_{\perp}$ with $N$ cannot be described in terms of a general function, but seems to be dependent on parameters like the electric field strength (cf. Table 1). Final conclusions on such details require more simulations and an increased accuracy of the results.

As shown previously, our present simulations demonstrate field-induced dissociation of counterions from the ion atmosphere of the polymer and also saturation of field-induced dipole moments. Saturation of dipole moments appears to be a consequence of field-induced ion dissociation.

A major advantage of the Brownian dynamics approach is the fact that time constants are obtained without problems. The magnitude of the simulated dipole rise-time constants and the absence of a chain length dependence are in close agreement with experimental data derived from dichroism rise curves measured for DNA restriction fragments. ${ }^{37}$ The experimental data do not provide any evidence for an overshoot of the dipole moment shortly after field application. Information about the existence of an overshoot effect can hardly be obtained from the experimental data, because the polarization has not been observed directly but indirectly via a delayed onset of the rotation of the DNA fragments into the direction of the electric field. The absence of any detectable chain length dependence in the polarization time constants suggests that the essential step in the polarization mechanism does not correspond to motion of ions along the polymer as implied in many theories, but corresponds more closely to biased dissociation as implied in the mechanism proposed on the basis of the experimental data. ${ }^{37}$

Most of our present data were simulated for polyions without added salt or with relatively low concentrations of added salt, in order to limit the amount of computer time required for these simulations. Our data obtained for a polyion with 40 residues at different salt concentrations indicate a strong increase of the contribution from hydrodynamic interactions with increasing salt concentration. Moreover these data indicate a rather strong increase of the dipole moment with increasing salt concentration, which is not consistent with experimental data. ${ }^{10,11}$ Results obtained by some other theoretical models also provided an increase of the dipole moment with the salt concentration (cf. refs 39 and 40). The clear disagreement of theoretical and experimental results indicates a problem. In our present simulations we have included all the important contributions to the overall dipole induced in a polyion and its ion atmosphere. However, our present simulation procedure still represents an approximation, because we have not yet simulated the torque experienced by rodlike polyelectrolytes under the influence of external electric fields. Thus, the next step should be simulation of the field-induced rotation of polyelectrolyte rods into the direction of the field vector and calculations of the change of the optical anisotropy resulting from the rotation. This type of simulation clearly requires larger amounts of computer time than that described above.

Acknowledgment. We are indebted to Dr. Ulrich Schwardmann for an efficient optimization of the algorithms used in our simulations and for implementation of our program on a parallel computer. We used the facilities of the Gesellschaft für wissenschaftliche Datenverarbeitung $\mathrm{mbH}$, Göttingen, for our simulations.

\section{References and Notes}

(1) Schwarz, G. Z. Phys. 1956, 145, 563.

(2) Schwarz, G. Z. Phys. Chem. N. F. 1959, 19, 286

(3) Schwarz, G. J. Phys. Chem. 1962, 66, 2636.

(4) Mandel, M. Mol. Phys. 1961, 4, 489.

(5) Oosawa, F. Biopolymers 1970, 9, 677.

(6) Rau, D. C.; Charney, E. Biophys. Chem. 1981, 14, 1.

(7) Manning, G. Q. Rev. Biophys. 1978, 11, 179.

(8) Hogan, M.; Dattagupta, N.; Crothers, D. M. Proc. Natl. Acad. Sci. U.S.A., 1978, 75, 195 .

(9) Fixman, M.; Jagannathan, S. J. Chem. Phys. 1981, 75, 4048.

(10) Stellwagen, N. Biopolymers 1981, 20, 399.

(11) Elias, J. G.; Eden, D. Macromolecules 1981, 14, 410.

(12) Diekmann, S.; Hillen, W.; Jung, M.; Wells, R. D.; Porschke, D. Biophys. Chem. 1982, 15, 157.

(13) Porschke, D. Biopolymers 1989, 28, 1383.

(14) Porschke, D. Biophys. Chem. 1985, 22, 237

(15) Szabo, A.; Haleem, M.; Eden, D. J. Chem. Phys. 1986, 85, 7472.

(16) Mandel, M.; Odijk, T. Annu. Rev. Phys. Chem. 1984, 35, 75

(17) Charney, E. Q. Rev. Biophys. 1988, $21,1$.

(18) Kikuchi, K.; Yoshida, M.; Maekawa, T.; Watanabe, H. Colloid and Molecular Electro-Optics 1991; Jennings, B. R., Stoylov, S. P., Eds.; Institute of Physics Publ.: Bristol, 1992; p 7.

(19) Watanabe, H.; Yoshida, M.; Kikuchi, K.; Maekawa, T. Colloid and Molecular Electro-Optics 1991; Jennings, B. R., Stoylov, S. P., Eds.; Institute of Physics Publ.: Bristol, 1992; p 61.

(20) Yoshida, M.; Kikuchi, K.; Maekawa, T.; Watanabe, H. J. Phys. Chem. 1992, 96, 2365.

(21) Grycuk, T.; Antosiewicz, J.; Porschke, D. J. Phys. Chem. 1994 98, 10881-10887.

(22) Mohanty, U.; Zhao, Y. Biopolymers 1996 38, 377-388.

(23) Ewald, P. P. Ann. Phys. (Leipzig) 1921, 64, 253.

(24) Ermak, D. L. J. Chem. Phys. 1975, 62, 4189.

(25) Happel, J.; Brenner, H. Low Reynolds Number Hydrodynamics; Noordhoff International Publishing: Leyden, 1973.

(26) Garcia de la Torre, J.; Bloomfield, V. A. Q. Rev. Biophys. 1981, $14,81-139$

(27) Ermak, D. L.; McCammon, J. A. J. Chem. Phys. 1978, 69, 1352.

(28) Rotne, J.; Prager, S. J. Chem. Phys. 1969, 50, 4831.

(29) Yamakawa, H. J. Chem. Phys. 1970, 53, 436-443.

(30) Antosiewicz, J.; Porschke, D. J. Biomol. Struct. Dyn. 1988, 5, 819837.

(31) Antosiewicz, J.; Porschke, D. J. Phys. Chem. 1989, 93, 53015305.

(32) Wien, M. Physik. Z. 1931, 32, 545.

(33) Wissbrun, K. F.; Patterson, A. J. Polym. Sci. 1958, 33, 235.

(34) Porschke, D. Annu. Rev. Phys. Chem. 1985, 36, 159.

(35) Onsager, L. J. Chem. Phys. 1934, 2, 599.

(36) Onsager, L.; Liu, C. T. Z. Phys. Chem. 1965, 228, 428.

(37) Porschke, D. Biophys. Chem. 1985, 22, 237-247.

(38) Porschke, D.; Antosiewicz, J. Biophys. J. 1990, 58, 403-411.

(39) Manning, G. S. J. Chem. Phys. 1993, 99, 477-486.

(40) Antosiewicz, J.; Porschke, D. J. Phys. Chem. 1991, 95, 59835988. 